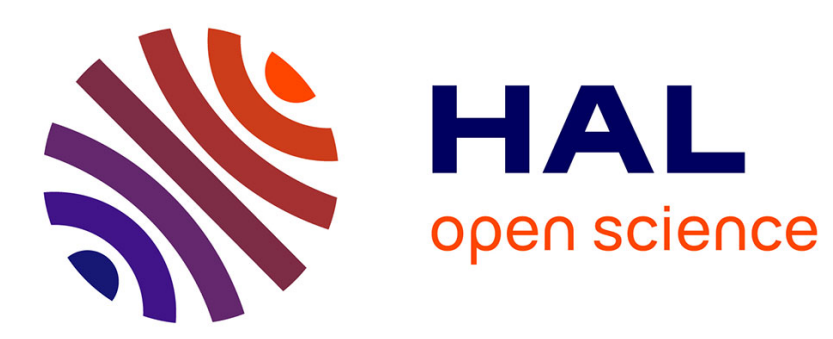

\title{
Des contrats de terroirs pour les Pays de la Gascogne gersoise
}

Olivier Dehoorne

\section{To cite this version:}

Olivier Dehoorne. Des contrats de terroirs pour les Pays de la Gascogne gersoise. Norois, 1999, 45 (181), pp.127-139. hal-01406645

\section{HAL Id: hal-01406645 \\ https://hal.science/hal-01406645}

Submitted on 1 Dec 2016

HAL is a multi-disciplinary open access archive for the deposit and dissemination of scientific research documents, whether they are published or not. The documents may come from teaching and research institutions in France or abroad, or from public or private research centers.
L'archive ouverte pluridisciplinaire HAL, est destinée au dépôt et à la diffusion de documents scientifiques de niveau recherche, publiés ou non, émanant des établissements d'enseignement et de recherche français ou étrangers, des laboratoires publics ou privés. 


\title{
Des contrats de terroirs pour les Pays de la Gascogne gersoise
}

\author{
Olivier DEHOORNE \\ Département de Gèographie, Université de Poitiers \\ 97, avenue du Pecteur Pneau, 86022 Poitiers Cadex
}

L dernier contrat de plan État-Région, le Conseil régional de Midi-Pyrénées a opté pour une procédure originale avee la mise en place des contrats de développement reposant sur une nouvelle dimension territoriale : le " terroir "

En fait, l'aménagement actuel de l'espace régional s'organise autour de trois démarches complémentaires donnant lieu à des contrats spécifiques en fonction des types de territoires. Le premier voler concerne la métropole toulousaine qui fair l'objet d'une contractualisation spécifique, Viennent ensuite les contrats d'agglomération qui concernent les villes moyennes de la région, c'est-à-dire les préfecrures des différents départements et quelques villes secondaires. Enfin, pour soutenir l'aménagement des campagnes et le développement de ces territoires, la Région met en place ces fameux contrats de terroirs.

Cette approche de l'aménagement régional est fondée sur les caractéristiques de MidiPyrénées: un espace dominé par une métropole dynamique, relayée par une modeste trame de villes moyennes et surtour marqué par l'importance des campagnes, encore largement orientées vers les activités agricoles. Ces zones rurales qui couvrent les neufdixièmes du territoire comptent $40 \%$ de la population régionale ; ces ruraux se répartissent entre 2800 communes dont $90 \%$ ont moins de 1000 habitants.

Conscients que l'émiettement des communes nécessite d'imaginer de nouvelles formes d'organisations intercommunales, des réflexions sont engagées aux niveaux régional et départementaux pour doter les campagnes d'outils adaptés aux politiques rurales. Au premier abord, il semble que la notion de Pays, mise en avant dans le cadre de la loi d'orientation pour l'aménagement et le développement du territoire du 4 février 1995, ne retienne pas l'attention des acteurs locaux - bien que deux territoires aient été retenus comme Pays de préfiguration : le Pays des Coteaux des HautesPyrénées er le Pays Bourian (Lor), parmi les quarante-deux sites retenus par le gouvernement (le 4 août 1995).

Les responsables régionaux privilégient le concept de " terroir " comme nouveau cadre territorial. Ce territoire - dont la teneur présente autant d'ambiguïtés que le Pays - est cependant privilégié en raison de sa dimension spatiale qui a immédiatement été présentée comme plus réduite que le Pays et donc plus proche des préoccupations des populations locales.

NOROIS, 1999, POITIERS, t. 45, $\mathrm{N}^{*} 181$, p. 127-139 
Cette nuance fut déterminante pour les départements les plus ruraux comme le Gers où $64,6 \%$ des 175000 habitants résident dans des communes rurales. Les initiatives intercommunales étaient des plus réduites au début de l'opération; par exemple le département du Gers ne comptait qu'une seule communauté de communes associant trois communes proches d'Auch. Le Gers est fortement marqué par la ruralité et ses habitants restent attachés aux structures communales et au rôle central de leur chef-lieu de canton.

Dans ce contexte, les contrats de développement de terroirs sont présentés comme complémentaires à la démarche des Pays proposés par l'État. Localement le Pays est perçu comme un "grand bassin de vie " tandis que le terroir, plus proche des réalités rurales, correspond au "bassin de vie quotidienne ". Soulignant la nécessité de s'engager dans l'intercommunalité tout en protégeant les ruraux d'un éclatement de leur cadre de vie, cette distinction d'échelle a suscité un réel intérêt dans les campagnes gasconnes. Cependant, peut-on envisager qu'à terme le regroupement de plusieurs terroirs puisse aboutir à la définition de Pays ? Sur le plan théorique, cette évolution peut se concevoir mais, dans la réalité, des associations intercommunales aux échelles supérieures apparaissent délicates, notamment dans le département du Gers.

\section{Des « terroirs " à la place des Pays ?}

Les bassins de vie définis par l'INSEE ont mis en évidence l'existence de nouveaux territoires fonctionnels organisés à partir du rayonnement de certaines communes centrales dotées d'équipements essentiels.

Pour déterminer ces nouvelles unités spatiales que sont les terroirs, la réflexion conduite sur les bassins de vie fut affinée en introduisant une appréciation relative à la fréquence de ces déplacements qui doivent être quotidiens. Ceci suppose donc des territoires pas trop vastes permettant d'aller aisément d'un point à l'autre de cet espace avec des temps de déplacement raisonnables, c'est-à-dire des trajets dont la durée n'excède pas la demi-heure.

Certes il faut atteindre certains volumes de population pour assurer le fonctionnement des équipements et des services en place, mais la prise en compte de la durée de ces déplacements est fondamentale dans la logique de construction des territoires par les populations résidentes.

Le contexte local, rural, reste largement imprégné du rythme des activités agricoles même si ces campagnes accueillent une proportion croissante de néo-ruraux, retraités ou actifs exerçant notamment dans les principaux centres urbains extérieurs au département. Cependant, que les populations soient natives de ces territoires ruraux ou qu'elles soient récemment installées, le nouveau cadre à partir duquel on souhaite créer, ou favoriser l'émergence, de ces bassins de vie quotidienne ne doit pas être trop grand.

Tout le monde s'accorde pour reconnaître l'échelon communal dépassé - bien qu'on en reconnaisse l'efficacité pour régler les problèmes de la vie quotidienne. Ces populations diverses s'entendent sur l'élargissement de cette structure de base, pour l'harmoniser au mieux avec leur espace vécu. Cet espace sort des limites du canton sans pour autant s'étendre inconsidérément. Les dimensions territoriales citées dans la majorité des cas concernent tout au plus trois ou quatre cantons. Ce cadre semble 


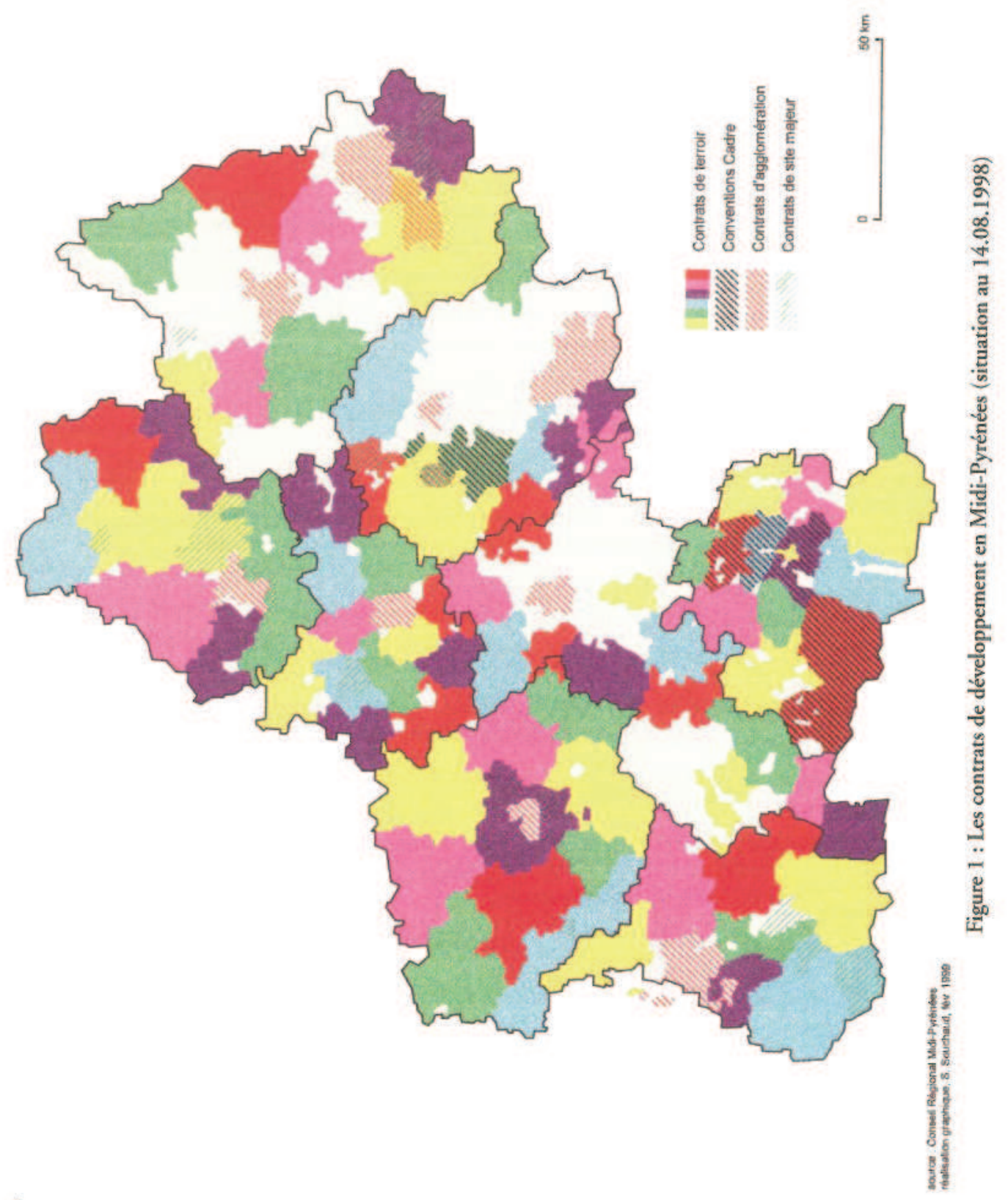


correspondre aux expériences vécues localement de l'espace rural. Les résidents le définissent par un sentiment de familiarité avec les lieux, les gens.

En fait ces territoires ne sont pas exactement des espaces vécus quotidiennement : les déplacements vers les principaux bourgs-centres y sont habituels et relativement fréquents. Ces espaces vécus sont définis par la présence de fonctions majeures qui concernent l'essentiel des populations locales.

Sur le plan commercial, les supermarchés suppléent l'appareil traditionnel déficient. Ils présentent un maillage cohérent, d'une trentaine d'unités sur l'ensemble du département, qui semble convenir aux attentes locales. Les hypermarchés ne sont pas des équipements indispensables à ces territoires, d'ailleurs seulement quatre communes en sont dotées : Auch (la principale ville du département), Eauze et Nogaro (deux chefslieux de canton de la partic nord-ouest du Gers) et Barcelone-du-Gers (commune limitrophe de la ville landaise d'Aire-sur-Adour).

Enfin, il convient de rappeler que ces espaces sont dotés de marchés notables qui tout en conservant des fonctions commerciales appréciables sont surtout des lieux de rencontre des populations du terroir.

Sur le plan scolaire, le collège figure parmi les équipements indispensables du terroir. Le lycée paraît plus lointain, réservé à des territoires plus importants. Par exemple la moitié occidentale du département ne compte qu'un seul lycée professionnel.

Avec le commerce et le système éducatif, le secteur de la santé est le troisième volet incontournable pour conduire cette réflexion territoriale. Dans ce département faiblement peuplé, le poids croissant de l'économie liée au troisième âge permet d'avoir un territoire relativement bien doté. Huit hôpitaux locaux (anciennement des hospices) relayés par des maisons de retraite médicalisées constituent autant de services de proximité permettant de définir les contours des terroirs.

La mobilité des populations rurales évolue. Une part notable des actifs effectue des déplacements importants hors de leur espace de résidence, vers Auch ou des villes extérieures. Les populations concernées admettent qu'il peut y avoir dissociation entre le territoire de résidence, où l'on s'implique dans le relationnel et les divertissements, et le lieu d'exercice de l'activité professionnelle.

En dépit de ces mobilités accrues, les résidents sont attachés à l'identité de leur terroir qu'ils contribuent à perdurer et à renouveler comme à travers des fêtes locales, des grandes manifestations estivales ou plus simplement les rencontres du rugby. Autant de rituels qui permettent aux résidents de vivre cet espace, de sentir qu'un territoire est partagé par une communauté d'individus aux intérêts communs. En ce sens, les nombreux festivals - dont certains participent à la notoriété du Gers - contribuent à mobiliser les populations locales tout au long de l'année.

En dépit de la mobilité, de l'arrivée de nouvelles populations, la convergence des résidents vers les communes centrales les mieux équipées et leur participation aux manifestations locales - comme acteurs ou comme spectateur - donnent naissance à un sentiment d'appartenance pour les populations les plus anciennement installées et sans doute de familiarité pour les derniers arrivés. Toutes ces réalités parfois abstraites doivent être évaluées pour envisager l'adhésion à la présentation de nouveaux cadres territoriaux. 


\section{Les oppositions entre terroir et Pays?}

Compte tenu des caractéristiques de ces espaces ruraux, il existe localement de réelles réticences à la notion de Pays telle qu'elle a été présentée sur la scène nationale. Ces territoires pertinents devraient selon les différents chiffres avancés comprendre entre 20 000 et 400000 habitants avec une moyenne autour de 70000 . Ces regroupements pourraient s'étendre sur une centaine de communes et une dizaine de cantons.

Ces différentes annonces signifieraient pour un département rural comme le Gers qu'il faudrait s'entendre sur la définition de trois pays. On imagine assez mal les limites que pourraient avoir de tels ensembles qui entraîneraient une perte de repère pour la majorité de la population et donc un manque d'intérêt pour des projets conduits à cette échelle.

Bien que le département compte trois arrondissements, il est également difficile d'envisager de les reprendre dans le cadre de "pays-arrondissement ", Les arrondissements gersois se présentent sous la forme de trois bandes successives dont les extrémités sont parfois distantes de plus de cent kilomètres. Lattractivité des souspréfectures est insuffisante et les arrondissements sont loin de correspondre aux bassins de vie.

Sans doute pour se prémunir de tout projet de découpage de telle dimension, les élus ont rapidement adopté les " terroirs ", ces unirés territoriales plus réduites mais mieux adaptées à leur espace vécu et à leurs préoccupations. Ces territoires relativement solidaires doivent permettre d'envisager des politiques soutenues par la base. Cette adhésion est indispensable pour la viabilité de ces ensembles et surtout pour envisager la mise en place de nouveaux processus de développement local qui est un objectif fondamental dans la réflexion sur les nouveaux découpages spatiaux.

\section{Le découpage des terroirs gersois}

La délimitation des territoires faisant l'objet de contrat de développement de terroir traduit une nouvelle approche de l'espace rural.

Dans ces campagnes, l'organisarion des grandes unités de relief, les pratiques agricoles plus ou moins adaptées aux spécificités du milieu naturel ont permis d'entreprendre des découpages relativement cohérents (fig. 2). Aujourd'hui, malgré la vocation agricole dominante de ces espaces - un quart des actifs du département travaille dans le secteur primaire -, les régions agricoles paraissent obsolètes. Si leurs paysages conservent quelques spécificités comme les vignobles de l'Armagnac ou les légumes de la Lomagne, l'homogénéisation des pratiques agricoles tend à uniformiser ces campagnes. Les céréales couvrent entre 40 et $60 \%$ de la S.A.U. selon les secteurs. Les vignobles ont considérablement régressé dans l'ouest du département tout comme les élevages bovins qui caractérisaient davantage les paysages de l'Astarac et du Haut-Armagnac. A l'inverse, l'aviculture progresse sur l'ensemble du Gers notamment avec les canards gras (deuxième département producteur derrière les Landes) et les oies grasses (troisième rang national). 


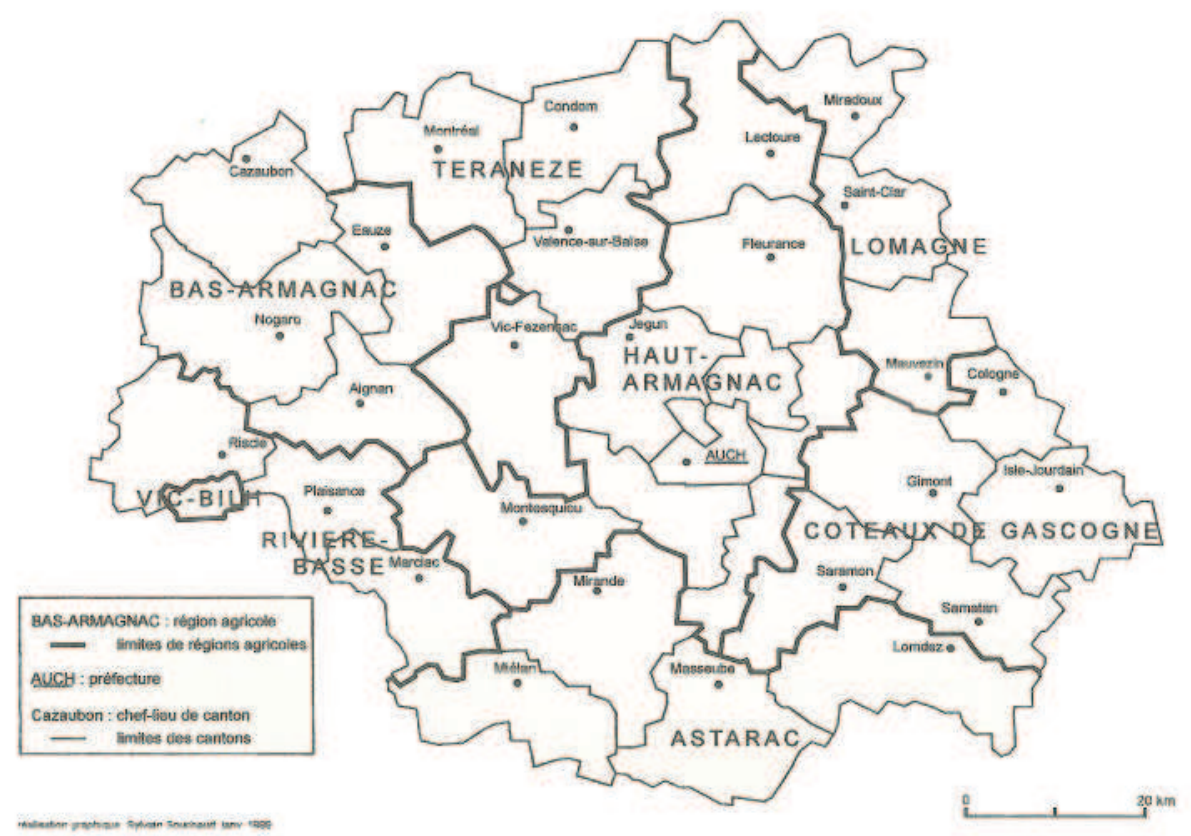

Figure 2 : Les régions agricoles du Gers.

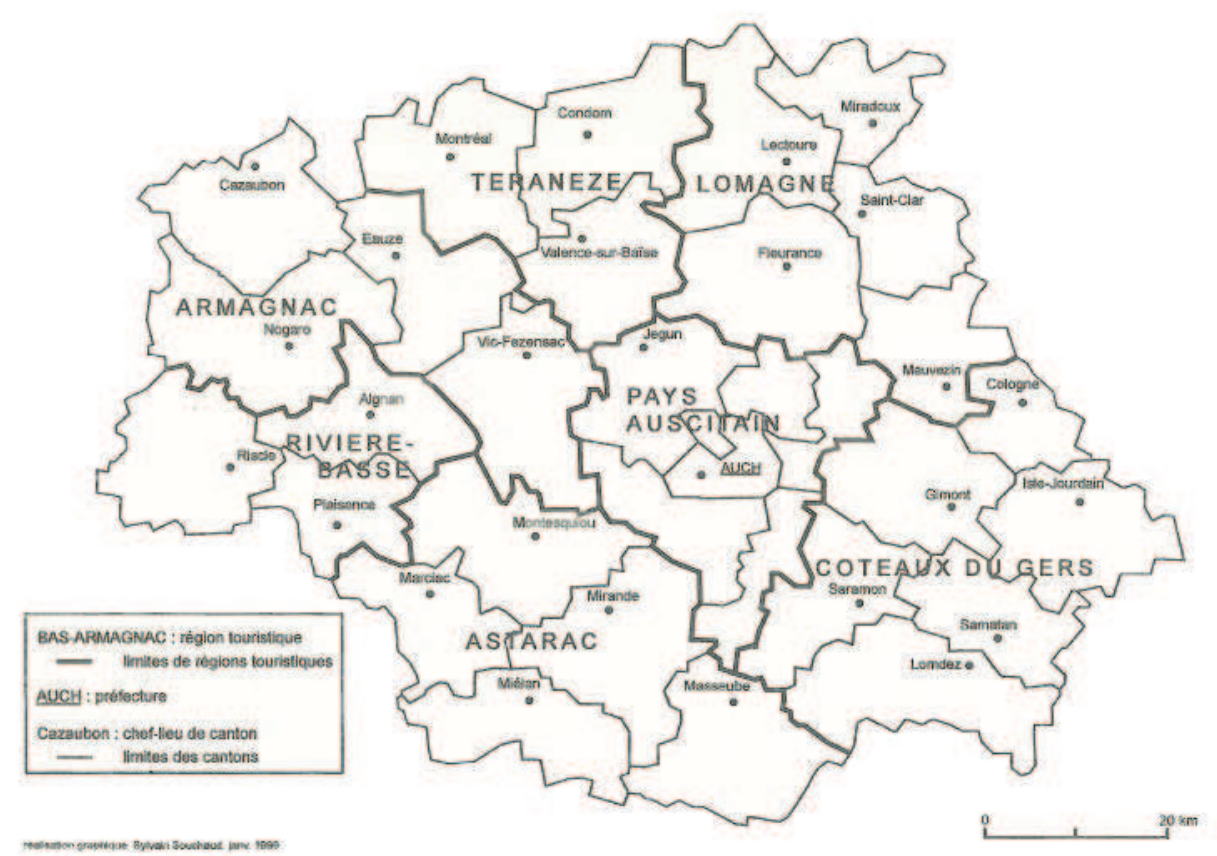

Figure 3 : Les régions touristiques du Gers. 
Les nouveaux découpages introduits par les responsables touristiques du département reposent en partie sur ces régions agricoles (fig. 3). Néanmoins quelques particularités, comme la création du Pays Auscitain, le glissement de l'Astarac vers la Rivière-Basse et la remontée de celui-ci sur l'Armagnac, s'expliquent par la recherche de territoires fonctionnels sur le plan touristique, présentant des superficies assez proches.

En fait, toutes ces approches sectorielles aboutissent à une série de découpages spécifiques avec les propres logiques. Les coopérations intercommunales sont des plus lentes à se mettre en place dans un tel contexte. Afin d'éviter cet écueil, les territoires définis dans le cadre de ces nouveaux contrars s"inscrivent dans une approche globale du territoire : ces espaces qui correspondent à des logiques de mobilité des populations résidentes doivent aussi prendre en compte les réalités agricoles comme les projets touristiques.

En accord avec le Conseil général et le Conseil régional, dix terroirs ont vu le jour dans le Gers. Dès juillet 1996, les trois premiers contrats sont signés avec le Terroir de d'Artagnan qui associe trois cantons périphériques du Bas-Armagnac, de la Ténazèze et du Haur-Armagnac. Le Terroir du Haut-Astarac, qui s'organise autour des cantons de Masseube et de Saramon, a attiré des communes des cantons de Lombez, Samatan et Auch-Sud-Est. Quant au Terroir de Val-de-Baïse-Armagnac, il correspond à l'ancienne région de la Ténarèze (fig. 4).

L'année suivante quatre autres terroirs sont reconnus : ceux de Lomagne (quatre cantons), de la Gimone et de l'Arrats qui s'est créé à partir de trois cantons périphériques de la Lomagne et des Coteaux de Gascogne. Le Terroir d'Adour-Gascogne qui s'organise à partit des vallées de l'Arros et de l'Adour correspond à la partie gersoise du bassin versant de l'Adour. Enfin le Terroir du Cocur de Gascogne regroupe les cantons d'Auch et de Jégun, mais la ville d'Auch qui fair l'objet d'un contrat de ville moyenne en est exclue. Cela n'est pas sans soulever des interrogations à propos de la cohérence de cet espace auquel on a soustrait la commune centrale qui l'organise.

Les deux derniers contrats n'ont été signés qu’en 1998. Ils concernent le Mirandais et le Val-de-Save qui est probablement l'un des moins homogènes, réunissant les communes ne faisant partie ni du terroir du Haut-Astarac ni de celui de la Gimone et de l'Arrats.

Les dix terroirs du Gers regroupent entre trois et quatre cantons pour un total de 40 à 70 communes (avec un maximum de 73 communes pour l'Adour-Gascogne). Leur poids démographique est compris entre 10000 et 22000 habitants avec des superficies allant de 500 à $800 \mathrm{~km}^{2}$; le terroir le plus peuplé étant celui de la Lomagne avec 21152 habitants répartis sur $809 \mathrm{~km}^{2}$. Les densités les plus faibles appartiennent aut Haut-Astarac $\left(16,8 \mathrm{~h} / \mathrm{km}^{2}\right)$ et au Pays de d'Artagnan $(17,5)$. Les autres présentent des densités proches de celle du département $\left(28 \mathrm{~h} / \mathrm{km}^{2}\right)$ (fig. 5$)$.

Le terroir du Mirandais qui correspond au seul canton de Mirande se distingue par ses dimensions : 7468 habitants pour 23 communes mais avec une densité de $28,1 \mathrm{~h} / \mathrm{km}^{2}$.

Bien que ces territoires soient reconnus dans leur globalité, certains points doivent être évoqués. 


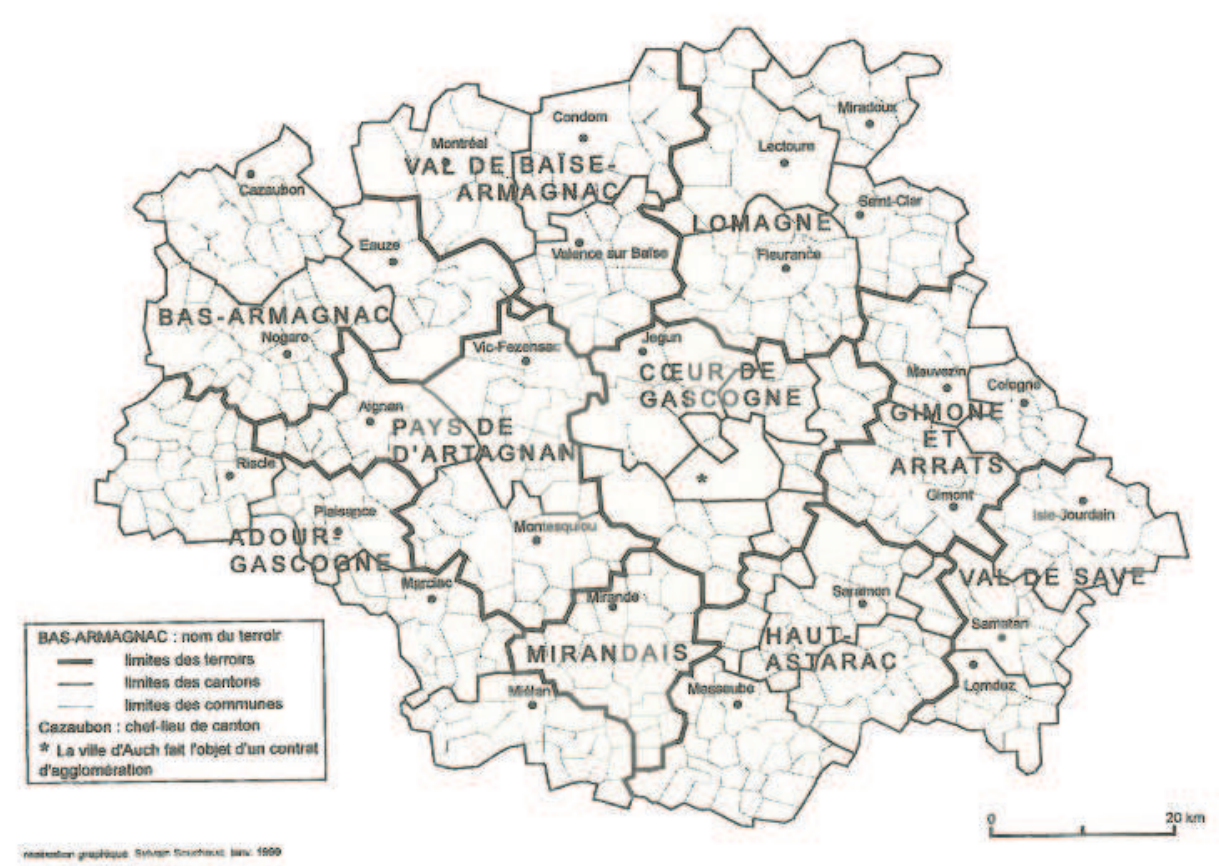

Figure 4 : Les contrats de terroirs du Gers.

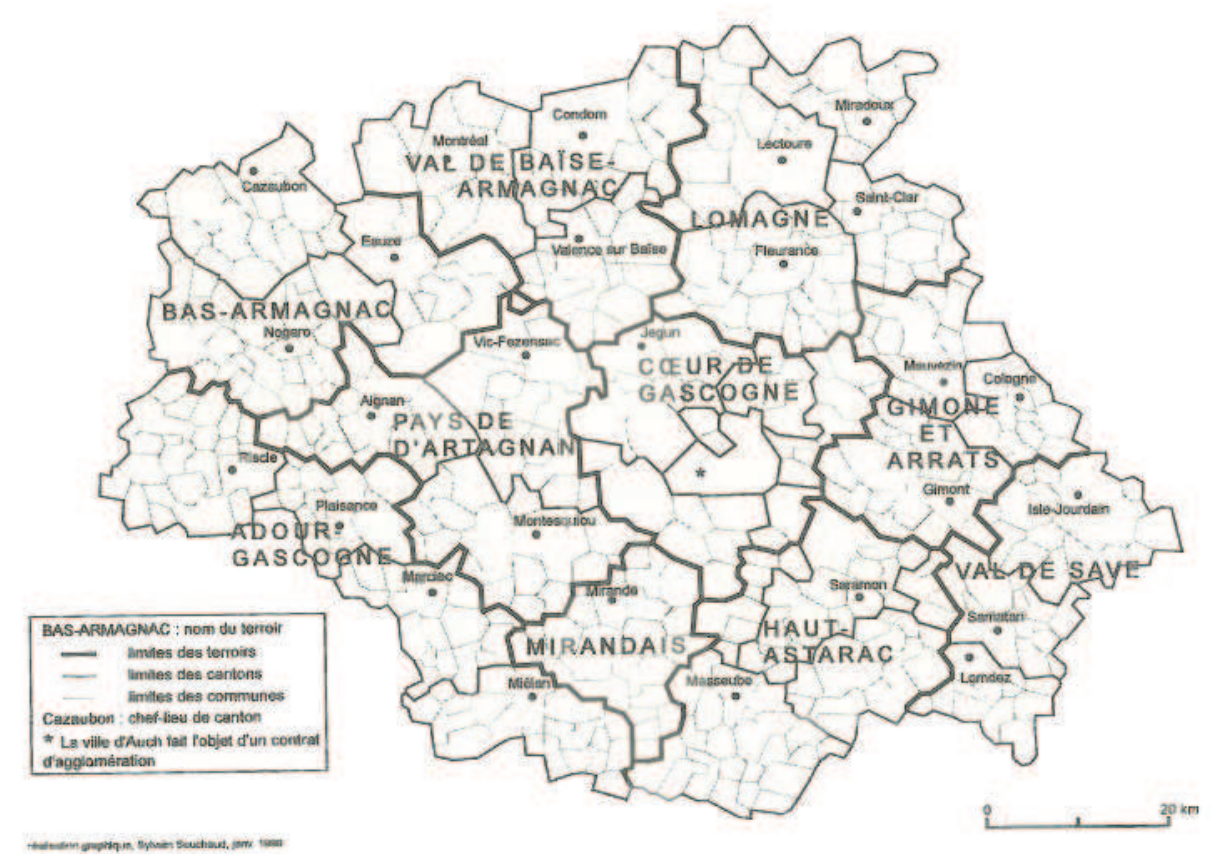

Figure 5 : Les peuplements des terroirs gersois. 
En premier lieu se pose le problème des limites départementales qui ont marqué les frontières extérieures de ces terroirs. Or les bassins de vie quotidienne sortent parfois de ce cadre : les collines du Bas-Armagnac glissent vers la plaine landaise et ses populations ne sont pas insensibles à l'attraction de communes landaises comme Gabarret et Airesur-Adour, privilégiant même la ville de Mont-de-Marsan au détriment de la préfecture gersoise.

Dans le sud-est du département, l'éclatement de certains cantons dans le secteur du Val-de-Save et du Haut-Astarac traduit une certaine rupture qui se dessine dans le tissu rural. Le Val-de-Save correspond à un secteur largement influencé par Toulouse. C'est ainsi que $30 \%$ des actifs du canton de l'Isle-Jourdain travaillent dans cette agglomération. A l'opposé, dans le Haut-Astarac, les agriculteurs représentent $45 \%$ de population active.

A l'intérieur même des cantons de Samatan et de Lombez, les communes se sont partagées entre ces deux terroirs, celles qui ont choisi le Haut-Astarac correspondant à l'ancien canton de Simorre disparu dans les années 1950. Cette sécession dans le cadre des terroirs s'est même accompagnée de demandes de reconstitution de l'ancien canton.

A l'image des rivalités qui existent sur le plan sportif, avec les principaux clubs de rugby et les terroirs ruraux qui les soutiennent, ces projets d'élargissements territoriaux butent sur des associations impossibles à réaliser localement. Certes, il $\mathrm{y}$ a parfois le jeu des élus locaux avec leurs diverses ambitions mais souvent ces antinomies reflètent des volontés largement relayées par les populations résidentes.

Au-delà de la disposition du relief, des héritages de l'histoire, des fortes empreintes agricoles, ces découpages traduisent la prise en compte des nouvelles logiques des mobilités rurales.

Avec près de $29 \%$ de sa population âgée de soixante ans et plus, ce département est parmi les plus vieillis de France. Cependant son niveau de peuplement se stabilise grâce aux gains enregistrés par le solde migratoire depuis une vingtaine d'années (les bilans des deux dernières périodes intercensitaires laissant apparaître des excédents supérieurs à 2000 personnes). Les nouvelles unités territoriales que sont les terroirs doivent donc favoriser l'insertion de ces arrivants, qu'ils soient retraités ou actifs travaillant dans les départements limitrophes. L'accroissement de ces flux quotidiens vers les foyers extérieurs risque d'aboutir à un écartèlement du Gers : les cantons périphériques constituant des marges rurales fragiles (depuis Cazaubon dans le nord-ouest jusqu’à Samatan dans le sud-est du département, les bourgs les plus peuplés atteignent à peine 1800 habitants), largement soumises aux influences des bassins d'activités limitrophes (fig. 6).

Certes les nuances de l'identité gasconne se sont toujours dessinées au contact de ces influences voisines (landaise, pyrénéenne...). Mais dans ce contexte il apparait essentiel que le terroir soit un espace de cohésion, un cadre privilégié qui puisse mobiliser les populations résidentes autour de projets. 


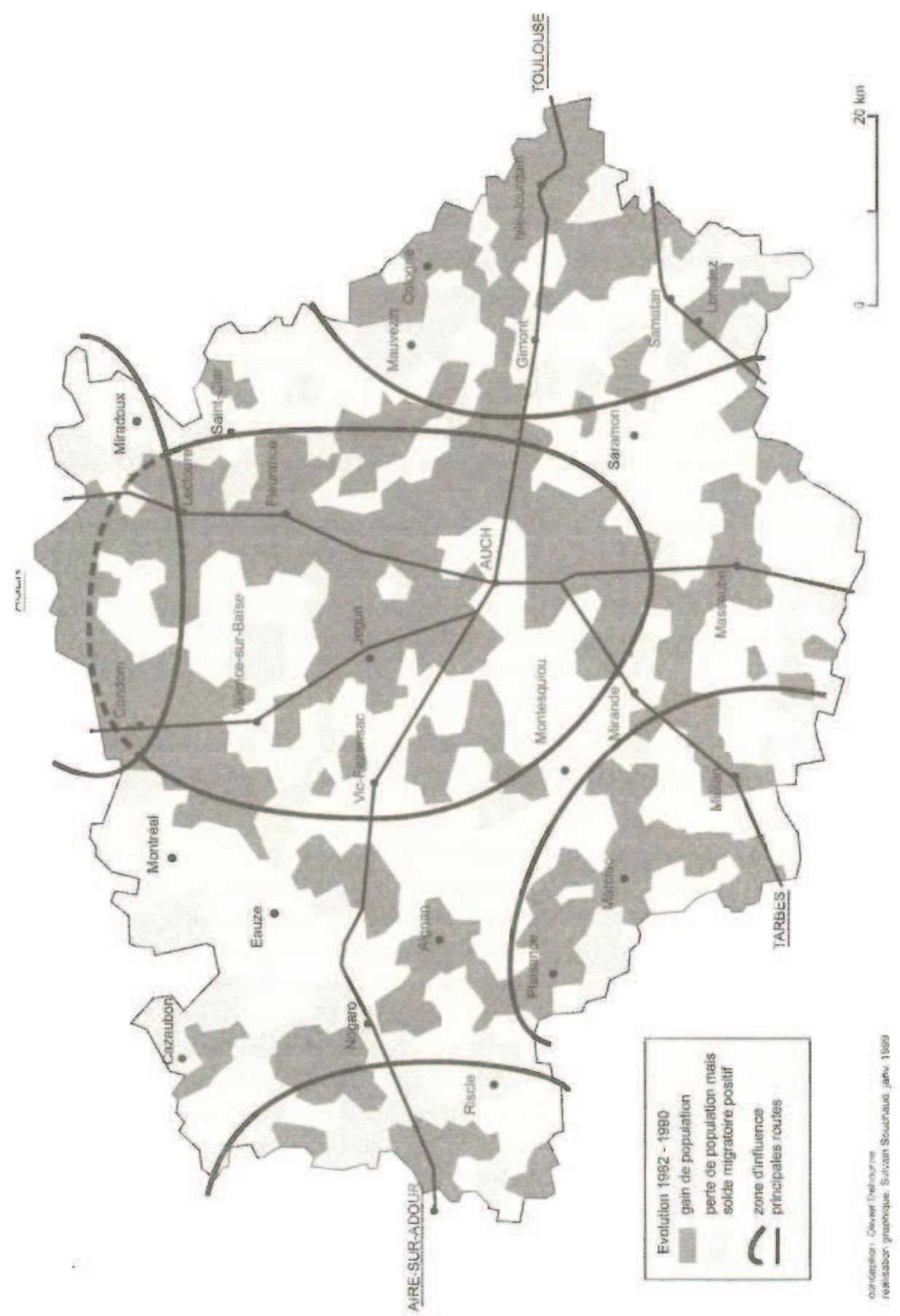

Figure 6 : Vers une réorganisation de l'espace départemental. 


\section{Des territoires de projets}

Dès 1995, les dix territoires correspondant aux différents bassins de vie quotidienne sont délimités. Ils constituent les cadres privilégiés de réflexion des élus et des acteurs locaux volontaires qui définissent leurs ambitions de développement. Le Département, par le biais de chargés de mission de l'Agence de Développement Économique, conseille et soutient les démarches locales. Ces projets doivent être mobilisateurs et générer des activités. La création d'emplois est essentielle pour que le projet soit accepté par la Région et le Département (le Conseil général s'est engagé à créer et financer cinq cents emploi-jeunes). La reconnaissance des projets donne lieu à la signature d'un contrat de développement de terroir.

Les contenus des projets qui comportent quelques originalités selon les terroirs s'organisent autour de trois axes prioritaires :

- la promotion d'activités traditionnelles ou originales ;

- des préoccupations d'ordre social avec les services à la personne ;

- le tourisme et l'amélioration du cadre de vie en général.

Le développement économique se fonde largement sur les acrivités agricoles telle que la filière viticole pour le Val-de-Baïse-Armagnac, le Bas-Armagnac er l'Adour-Gascogne qui entend aussi mieux valoriser sa filière bois ( 450 actifs). Les démarches relatives à l'amélioration de la qualité priment comme dans l'Astarac avec de l'élevage bovin biologique ou le projet d'appellation d'origine contrôlée " foie gras d'oie du Ciers " à partir de l'oie de Masseube.

L'organisation des services de proximité est soutenue par le Départentent qui propose un dispositif général adopté dans chaque contrat de terroir.

Au premier rang des préoccupations, les services de proximiré pour les personnes dépendantes. Lobjectif est de favoriser le maintien à domicile des personnes âgées tout en évitant leur isolement. Cela passe par la livraison de repas chauds à domicile à partir de structures diverses comme des maisons de retraite (comme celle de Simorre), d'hôpitaux (Fleurance), de centre de rééducation (Saint-Blancard dans le Haut-Astarac), ou d'un Centre communal d'action sociale (lectoure).

Le second volet porte sur la mise en place d'un service de télé-assistance (ou " téléalarme ") avec la diffusion d'un pendenrif qui permet à la personne âgée, sur simple pression, de demander de l'aide dans un établissement médicalisé du terroir qui assure une permanence. Les politiques envers les personnes âgées se poursuivent avec le développement des "auxiliaires de vie à domicilen, pour aider les personnes dépendantes dans leurs tâches quotidiennes, et d'autres systèmes d'entraide vont jusqu'au placement des personnes dépendantes dans le cadre de familles d'accueil agréées.

Le renforcement des services de proximité concerne aussi les transports à la demande - qui fonctionnent déjà depuis une quinzaine d'années en Lomagne -, le financement de commerces multi-services notamment dans le Bas-Armagnac (sur les communes de Laujuzan, Courrensan et Monguilhem) ou encore une halte-garderie itinérante dans le terroir de l'Adour-Gascogne (un bus équipé, avec un personnel qualifié, parcourt les communes du terroir pour rejoindre une salle d'accueil mise à la disposition des enfants). 
Tous les terroirs ont élaboré leurs projets de développement touristiques. L'un des plus porteurs pour le Gers concerne la remise en navigabilité de la Baïse (Val-de-BaïseArmagnac). Le Bas-Armagnac privilégie un axe touristique autour de la station de Barbotan-les-Thermes et du lac de l'Uby avec l'aménagement d'un centre de loisirs aquatiques. Le tourisme lié à l'eau est également à l'honneur dans l'Adour-Gascogne avec la recherche de complémentarité entre les quatre bases de loisirs actuelles (Marciac, Miélan, Plaisance, Riscle). Dans ce dernier terroir le tourisme culturel occupe une place importante avec les festivals (le jazz à Marciac, les orgues à Plaisance), les courses de vaches landaises (six arènes) ou la restauration des moulins de la Rivière-Basse.

Quelques orientations paraissent dominantes selon les programmes, elles n'en sont pas moins complémentaires. L'objectif étant un développement global du territoire. L'exemple du Haut-Astarac est significatif, le projet repose sur trois orientations majeures interdépendantes: l'agriculture, l'environnement et le tourisme. La qualité de l'environnement est considérée comme la première richesse de ce terroir, les-différentes activités économiques doivent donc valoriser cet aspect tout en le préservant. Cela passe par des pratiques agricoles moins polluantes notamment avec la mise aux normes des élevages. Une douzaine " d'emplois verts " sont créés pour veiller à la qualité des rivières et entreprendre des travaux paysagers, depuis le reboisement des collines avec des essences adaptées jusqu'à la réhabilitation des bâtis avec la recherche de matériaux typiques. En liaison avec les établissements scolaires apparaît une nouvelle formation orientée vers l'agriculture et l'environnement.

Sur le plan touristique, le Haut-Astarac a repris un projet autour de la chasse qui fut initié dans le cadre de la procédure LEADER. Le contrat de terroir permet de donner un nouvel élan à ce programme pilote. ${ }^{1}$

\section{Les terroirs, un premier pas vers les Pays}

Dans un premier temps, afin de bien souligner leur opposition au concept de Pays, les différents responsables et élus ont insisté sur le fait qu'il s'agissait de terroir et non de Pays. Tous ces contrats de développement concernent des terroirs, souvent avec l'adoption de nouvelles appellations.

Toutefois, des " associations de pays " sont créées pour assurer la mise en place et la conduite des contrats de terroirs. Ces associations couvrent les mêmes espaces que les terroirs et portent les mêmes noms. Sans doute s'agit-il d'une finesse destinée à rassurer à la fois les populations rurales hostiles à a ces pays trop vastes n et l'application de la loi relative aux Pays.

1. 11000 hectares répartis sur onze communes sont organisés en Groupement d'Intérêt Agro-SylvoCynégétique dont l'objectif est de proposer une politique touristique novatrice en relation avec la chasse. Ce projet propose notamment des formules de séjour comportant un habitat approprié et l'accompagnement systématique par des chasseurs locaux. 
Aujourd'hui, alors que ces nouveaux découpages territoriaux sont reconnus, que les projets des différents terroirs sont engagés, les élus se plaisent à rapprocher les termes de Pays et de terroir, les utilisant indifféremment. On est ainsi passé progressivement des terroirs aux Pays mais ces territoires présentés comme des Pays ont conservé des dimensions plus réduites, adaptées aux exigences d'un contexte rural dépourvu de trame urbaine significative.

Les terroirs cèdent la place aux nouveaux Pays, des " pays sans ville " pour la plupart si on considère que la ville commence à être significative à partir de 5000 habitants. Même si les volumes de populations ne sont pas très élevés, les dimensions de ces territoires disposant de dessertes essentiellement secondaires ne semblent guère augurer de nouveaux élargissements territoriaux. Par ailleurs, ces jeunes structures intercommunales paraissent déjà lourdes à gérer en raison du nombre de communes impliquées.

Cependant, à l'échelle de la région Midi-Pyrénées, la situation évolue davantage. Dès le début de 1998, huit terroirs se sont regroupés pour former trois Pays : deux dans l'Ariège (le Pays du Couserans et le Pays du Val d'Ariège) et le dernier dans le Tarn (le Grand Gaillacois).

\section{Conclusion}

La politique des contrats de développement de terroir a donné un nouvel élan à l'intercommunalité notamment dans un département à fort caractère rural et agricole comme le Gers. Cette situation devrait permettre de redistribuer avec plus d'efficacité les financements publics, privilégiant des projets de développement global des territoires et non des visions sectorielles.

Les terroirs semblent être une étape transitoire vers les Pays soit par l'union de plusieurs terroirs, soit par une dérive sémantique amenant à une substitution d'un terme par l'autre sans qu'il y ait extension de ces espaces. Les volumes démographiques peuvent alors être présentés comme insuffisants mais cette considération est nuancée par les superficies de ces territoires et les effectifs de communes concernées.

Cette politique va se poursuivre jusqu'à la fin du millénaire, ensuite les derniers changements de majorité intervenus à la tête de la région et de départements comme le Gers pourraient s'accompagner de nouvelles orientations. Les acteurs locaux ont le sentiment de vivre les amorces d'une période de transition qui pourrait être des plus intéressantes mais reconnaissent avancer dans un grand flou, tant aux niveaux local que national. Si les souhaits vont dans le sens d'un renforcement de ces nouveaux territoires d'action, se posera alors l'incontournable problème de la refonte des territoires d'élection.

\section{Bibliographie}

H.E.R.G.E.S., 1993. - L'Armagnac, Un produit. Un pays, Presses Universitaires du Mirail, Collection État des Lieux, Toulouse, 191 p.

INSEE, 1997. - Le Gers : l'économie de la tradition, INSEE Midi-Pyrénées, Toulouse, n83, 40 p. 